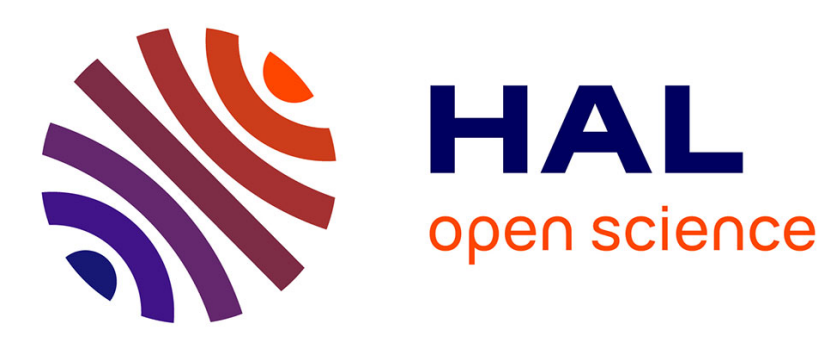

\title{
Characterization of ultimate bounds for systems with state-dependent disturbances
}

\author{
Sorin Olaru, Hiroshi Ito
}

\section{To cite this version:}

Sorin Olaru, Hiroshi Ito. Characterization of ultimate bounds for systems with state-dependent disturbances. IEEE Control Systems Letters, 2018, 2 (4), pp.797-802. 10.1109/LCSYS.2018.2849642 . hal-01830572

\section{HAL Id: hal-01830572 \\ https://hal-centralesupelec.archives-ouvertes.fr/hal-01830572}

Submitted on 5 Jul 2018

HAL is a multi-disciplinary open access archive for the deposit and dissemination of scientific research documents, whether they are published or not. The documents may come from teaching and research institutions in France or abroad, or from public or private research centers.
L'archive ouverte pluridisciplinaire HAL, est destinée au dépôt et à la diffusion de documents scientifiques de niveau recherche, publiés ou non, émanant des établissements d'enseignement et de recherche français ou étrangers, des laboratoires publics ou privés. 


\title{
Characterization of ultimate bounds for systems with state-dependent disturbances
}

\author{
Sorin Olaru ${ }^{1}$ and Hiroshi Ito $^{2}$
}

\begin{abstract}
This paper pursues a framework of set characterization of dynamical systems with state-dependent disturbances. It aims to propose a new approach to analysis and design of nonlinear systems involving non-differentiability and asymmetric components which hamper application and effectiveness of local linearization methods. Several characterizations of ultimate bounds are developed.The utility of shifting the fix point is formulated as a parametrization of the ultimate bounds.
\end{abstract}

Index Terms-Uncertain systems.

\section{INTRODUCTION}

The disturbance invariant sets for linear systems with static bounds on the additive disturbances have been characterized in the '90s [1], [2]. The particular set known as "minimal robust positive invariant set" received an important attention [3]-[5] with applications in several analysis and control design problems involving set-theoretic approaches [6], fault detection [7] or robust predictive control [8], [9] to mention only a few. The closely related concept of ultimate bounds [10] has been used for the characterization of dynamical systems in the presence of non-vanishing perturbation. It proved to be instrumental in the study of linear systems with bounded disturbances for at least two decades [11]. There are effective computational methods for the characterization of ultimate bounds for linear dynamics with (state-dependent) bounds for the disturbances [12], [13]. An interpretation of the ultimate bounds is given by the input-to-state stability (ISS) [14], [15].

The present paper deals with discrete-time nonlinear systems affected by disturbance inputs with state-dependent bounds. These disturbances are allowed to be arbitrary as long as they are contained in sets which are bounded point-wise. This includes asymmetrically non-Lipschitz state-dependent

This work was initiated during the visit of the first author at the Kyushu Institute of Technology. The support of the Matsumae International Foundation is gratefully acknowledged. The first author acknowledges the support via the CNCS - UEFISCDI grant, number PN-III-P1-1.1-TE-2016-0862, MOSCBIOS, within PNCDI III.

${ }^{1}$ Laboratoire des Signaux et Systèmes, CentraleSupélec, Université Paris Saclay, 3 rue Joliot Curie, 91190, Gif-sur-Yvette, France (sorin.olaru@centralesupelec.fr)

${ }^{2}$ Department of Systems Design and Informatics, Kyushu Institute of Technology, Iizuka 820-8502, Japan (e-mail: hiroshi@ces.kyutech.ac.jp) disturbance whose systematic treatment is non-trivial with the existing techniques.

Small-gain techniques are popular for verifying stability of equilibria of systems in the presence of state-dependent disturbances [16], [17]. However, their standard formulations by themselves do not readily provide sharp estimates of ultimate bounds. The main objective of the present paper is to put a light on the usefulness of numerical computations of ultimate bounds for linear systems with point-wise bounded sets of disturbances in the study of nonlinear dynamics. As expected, the linear part offers only local information on the ultimate boundedness. This paper presents a parametrization of these local bounds in terms of the linear dynamics, topology of the disturbance set and fixed-points, which will play an important role in the global analysis and design of nonlinear control systems.

\section{PRELIMINARY NOTIONS}

The Minkowski sum of two given sets $S, P \subset \mathbb{R}^{n}$ is described by $S \oplus P=\{x+y \mid x \in S, y \in P\}$. The norm of a finite dimensional vector $x \in \mathbb{R}^{n}$ is denoted $|x|$ and the epsilon ball centered at the origin is represented by $\mathbb{B}_{\epsilon}=$ $\left\{x \in \mathbb{R}^{n}:|x| \leq \epsilon\right\}$. The notation $\mathcal{P}\left(\mathbb{R}^{n}\right):=\left\{S \mid S \subset \mathbb{R}^{n}\right\}$ represents the power set of $\mathbb{R}^{n}$ in general, and $\mathcal{P}_{c l}\left(\mathbb{R}^{n}\right)$ is the set of all closed subsets of $\mathbb{R}^{n}$, including the empty set:

$$
\mathcal{P}_{c l}\left(\mathbb{R}^{n}\right):=\left\{S \in \mathcal{P}\left(\mathbb{R}^{n}\right) \mid S=\bar{S} \text { or } S=\emptyset\right\} .
$$

Definition 1 A set $S \subset \mathbb{R}^{n}$ is star-shaped at $\bar{x} \in$ $S$ if for any point $x \in S$ and $0 \leq \beta \leq 1$ it holds that $\beta x+(1-\beta) \bar{x} \in S$. The kernel of $S \subset \mathbb{R}^{n}$ denoted by $\operatorname{kern}(S)$ is defined as: $\operatorname{kern}(S)=$ $\{\bar{x} \in S: \beta x+(1-\beta) \bar{x} \in S, \forall x \in S, \beta \in[0,1]\}$.

Whenever the center $\bar{x}$ of a star-shaped set $S$ is relevant, the notation $S_{\bar{x}}$ is used. $\mathcal{S}_{\bar{x}}\left(\mathbb{R}^{n}\right)$ denotes the set of closed starshaped sets $S_{\bar{x}}$, containing $\bar{x}$ in their kernel. The Minkowski function associated to a set $S \in \mathcal{S}_{0}\left(\mathbb{R}^{n}\right)$ is defined as $\mathcal{H}_{S}(x)$ : $\mathbb{R}^{n} \rightarrow \mathbb{R}_{+}$with:

$$
\mathcal{H}_{S}(x)=\inf \left\{\alpha \in \mathbb{R}_{+}: x \in \alpha S\right\}
$$




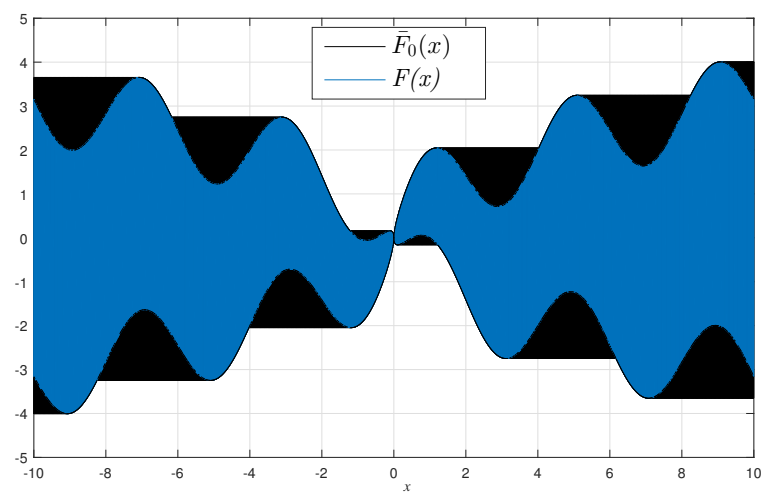

Fig. 1. Example of the set valued mapping $F($.$) in (3) and the increasing$ from 0 counterpart $\bar{F}_{0}($.$) constructed upon (2)$

Definition 2 A set-valued map $F: \mathbb{R}^{n} \rightarrow \mathcal{P}\left(\mathbb{R}^{m}\right)$ is called increasing from $\bar{x} \in \mathbb{R}^{n}$ if for all $x \in \mathbb{R}^{n}$ it holds that $F(\bar{x}+$ $\left.\lambda_{1}(x-\bar{x})\right) \subseteq F\left(\bar{x}+\lambda_{2}(x-\bar{x})\right)$ for $0 \leq \lambda_{1} \leq \lambda_{2}$.

Proposition 1 For any set-valued map $F: \mathbb{R}^{n} \rightarrow \mathcal{P}\left(\mathbb{R}^{m}\right)$, there exists a companion set-valued map increasing from $\bar{x} \in$ $\mathbb{R}^{n}$ defined as $\bar{F}_{\bar{x}}: \mathbb{R}^{n} \rightarrow \mathcal{P}\left(\mathbb{R}^{m}\right)$ with

$$
\bar{F}_{\bar{x}}(x)=\bigcup_{0 \leq \lambda \leq 1} F(\bar{x}+\lambda(x-\bar{x}))
$$

Example 1 Consider $\mathcal{F}: \mathbb{R} \rightarrow \mathcal{P}(\mathbb{R})$ defined by:

$$
F(x)=\left[-\sqrt{|x|}+\sin \left(\frac{\pi x}{2}\right), \sqrt{|x|}+\sin \left(\frac{\pi x}{2}\right)\right]
$$

The graph of $F($.$) as depicted in blue in Figure 1$ illustrates that this is not increasing from 0 . The union of the black and the blue envelopes presents the companion increasing setvalued map $\bar{F}_{0}($.$) derived from F($.$) based on (2).$

Consider a discrete-time system:

$$
x(k+1)=f(x(k))+w(k),
$$

where $x(k) \in \mathbb{R}^{n}$ is the state vector and $w(k) \in \mathbb{R}^{n}$ is a disturbance input at the time $k \in \mathbb{Z}_{+}$. The function $f: \mathbb{R}^{n} \rightarrow$ $\mathbb{R}^{n}$ admits the origin as equilibrium in the disturbance-free case $f(0)=0$.

Assumption 1 The system (4) is internally stable, in the sense that the only equilibrium is globally asymptotically stable in the disturbance-free case, i.e., whenever $w(k) \equiv 0, \forall k \in \mathbb{Z}_{+}$.

For the system (4), the signal $w(k) \in \mathbb{R}^{n}$ representing the additive disturbance is supposed to belong, at each sampling time, to a set described by a set-valued map: $w(k) \in W(x(k))$ with $W($.$) defined as W: \mathbb{R}^{n} \rightarrow \mathcal{P}_{c l}\left(\mathbb{R}^{n}\right)$ and satisfying the following point-wise boundedness:
Assumption 2 With the notation $\bar{w}(x)=\sup \{|w|: w \in$ $W(x)\}$, it is considered that $\bar{w}(x)<\infty$ for each $x \in \mathbb{R}^{n}$. For completeness, the following convention is used:

$$
\sup \{|w|: w \in W=\emptyset\}=0 .
$$

The point-wise boundedness excludes the case where $x(t)$ explodes in finite time. The following definition of boundedness will be used:

Definition 3 The solution $x$ of (4) is ultimately bounded ${ }^{1}$ if there exists a bounded set $S \subset \mathbb{R}^{n}$, possibly dependent on $x_{0}$, and a nonnegative integer $T\left(x_{0}, S\right)<\infty$, such that $x(k) \in S$ for all $k \geq T\left(x_{0}, S\right)$ and for all $x_{0} \in R^{n}$.

The paper proposes a method to compute ultimate bounds for the dynamics (4) and clarifies their properties in a set theoretic framework. In this context, it is important that the disturbance $w(k)$ is described by the state-dependent set, i.e., a set-valued map $W(x(k))$. This allows $w(k)$ to describe an endogenous signal in (4).

\section{NONLINEARITIES REPRESENTATION}

The theory of ultimate bounds for linear time-invariant (LTI) dynamical systems affected by point-wise bounded disturbances is relatively mature. The analysis of LTI systems prove to be used for the study of the general system (4) by starting from the simple observation that:

$$
\begin{aligned}
x(k+1) & =f(x(k))+w(k) \\
& =A x(x)+\underbrace{(f(x(k))-A x(k)+w(k)))}_{\tilde{w}(k)},
\end{aligned}
$$

with $\tilde{w}(k) \in \tilde{W}(k)$ which contains, on top of the additive uncertainty, the mismatch between the linear and the nonlinear model. This can be achieved via the transformation of the state-dependent disturbance:

$$
\tilde{W}(x(k))=W(x(k)) \oplus\{f(x(k))-A x(k)\} .
$$

Is worth to be mentioned that both $f($.$) and W($.$) can be$ asymmetric and non-Lypschitz and consequently this will be the case for the resulting mapping $\tilde{W}($.$) .$

Example 2 Consider the dynamics (4) with

$$
f(x)= \begin{cases}\frac{x^{3}-0.2 x(x-2)^{2}}{x^{3}-0.2(x-8)(x-2)^{2}} & \text { for } x \in[-3,3] \\ x / 2+3 & \text { for } x \notin[-3,3]\end{cases}
$$

\footnotetext{
${ }^{1}$ The attribute robustly is to be added to these notions if the properties hold for any $w \in W(x)$
} 


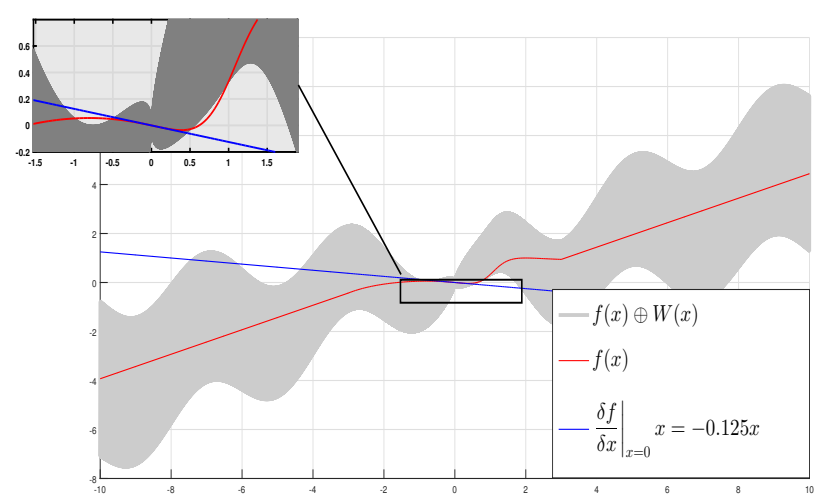

Fig. 2. Disturbance-free mapping vs. the mapping with uncertainty

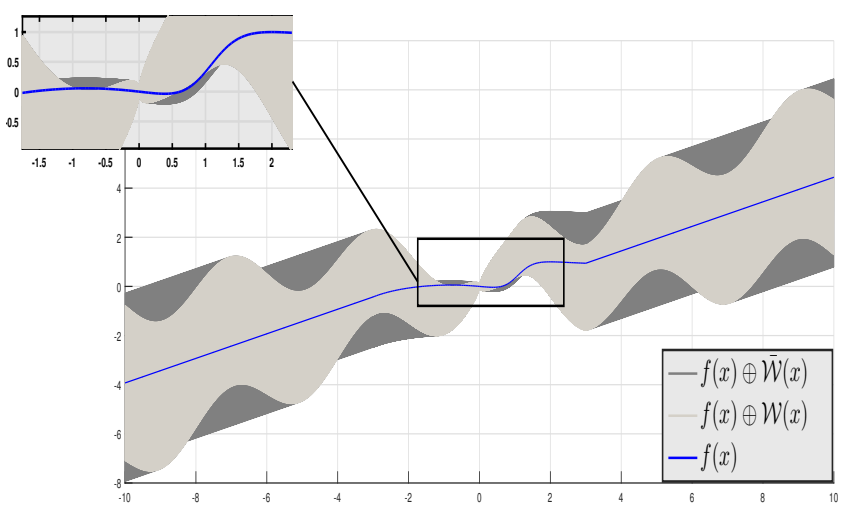

Fig. 3. Mapping with uncertainty vs. the increasing from 0 counterpart

and

$w(k) \in \underbrace{\left[-\sqrt{|x(k)|}+\sin \left(\frac{\pi x(k)}{2}\right), \sqrt{|x(k)|}+\sin \left(\frac{\pi x(k)}{2}\right)\right]}_{\mathcal{W}(x(k))}$,

corresponding to (3). Figure 2 shows that the nonlinear map $f(\cdot)+w(\cdot)$ in the neighbourhood of the origin substantially differs from the local linear approximation, i.e., the Jacobian of $f$ at zero. Figure 3 illustrates the uncertainty around the nonlinear model (4) as measured by the state-dependent bound and the increasing-from-0 counterpart. Figure 4 shows the poor global quality when Jacobian linearization is used for $A$ in (6). Interestingly, Figure 5 shows that choosing $A=0.5$, reduces the impact on the increasing-from-0 disturbance set. Despite the mismatch around the equilibrium, the uncertainty envelope associated with $A=0.5$ is sharp as shown in Figure 6.

This example illustrates that the matrix $A$ should not be conceived only as a local approximation (via the Jacobi linearization for example) of the (stable) nonlinear mapping

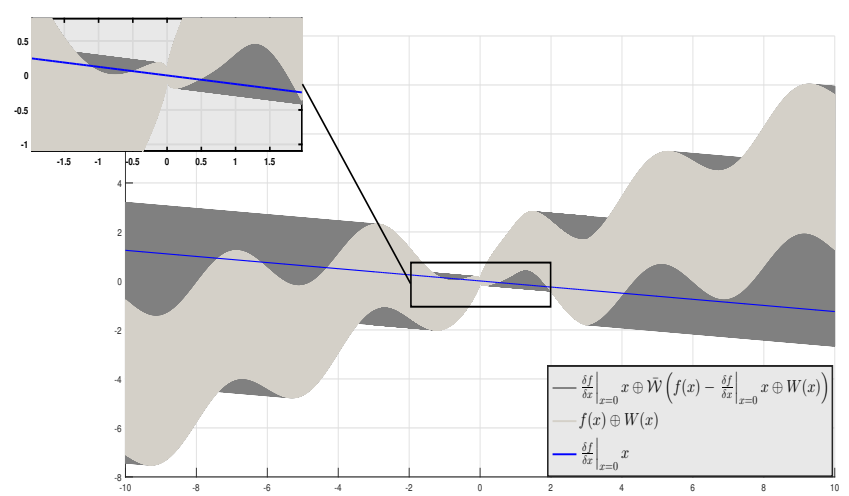

Fig. 4. Mapping using a Jacobi linearization vs. the increasing from 0 counterpart.

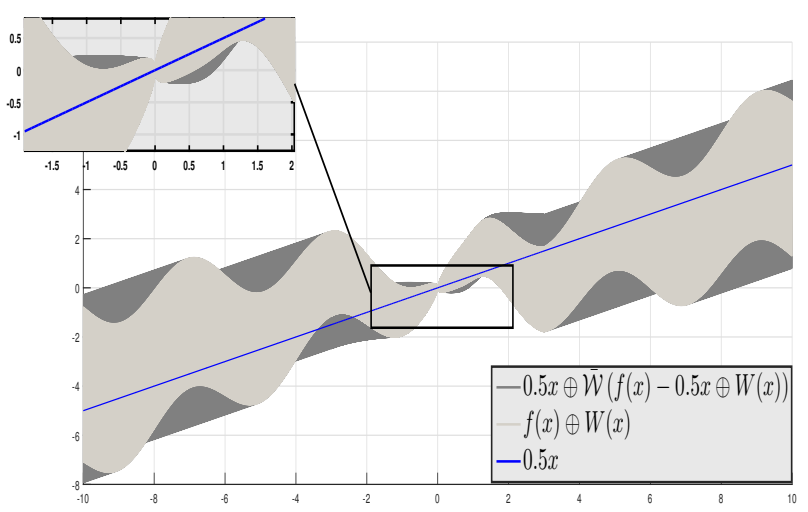

Fig. 5. Mapping using a linear approximation vs. the increasing from 0 counterpart.

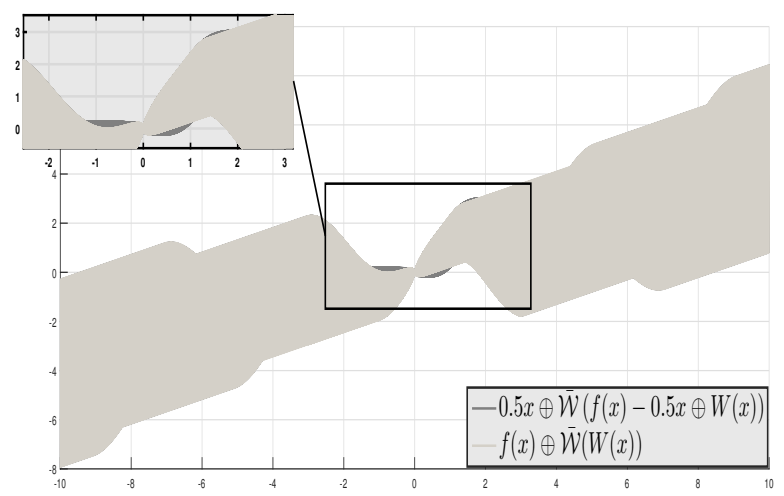

Fig. 6. Comparison of the set valued mapping using increasing from 0 set disturbances. Original nonlinear system vs. linear approximation of the dynamics. 
$f($.$) . The choice of the linear part represents an important$ degree of freedom which can be exploited as long as the model mismatch $\epsilon(x)=f(x)-A x$ is incorporated in the disturbance set for all $x \in \mathbb{R}^{n}$. The state-dependent bounding $\tilde{W}($.$) defined in (7) represents the envelope of the mismatch.$ The freedom of $A$ can be used to reduce globally the impact on the uncertainty.

\section{A. Linear systems with bounded additive disturbances}

Consider the linear system with additive disturbances

$$
x(k+1)=A x(k)+w(k),
$$

with $w(k) \in S$ with $S \in \mathcal{S}_{0}\left(\mathbb{R}^{n}\right)$, a star-shaped set at the origin and Assumption 1 holds true for $f(x(k))=A x(k)$.

For the dynamics (9) there exists an invariant $\operatorname{set}^{2}$ :

$$
\Omega_{A}(S):=\bigoplus_{i=0}^{\infty} A^{i} S
$$

containing a collection of initial states for which the future trajectories remain within the same set and which satisfy: $A \Omega_{A}(S) \oplus S=S$. Independent of the star-shaped properties of the argument, the relationship (10) defines a mapping

$$
\Omega_{A}(.): \mathcal{P}_{c l}\left(\mathbb{R}^{n}\right) \rightarrow \mathcal{P}_{c l}\left(\mathbb{R}^{n}\right) .
$$

Theorem 1 For any set $S \in \mathcal{S}_{0}\left(\mathbb{R}^{n}\right)$ there exists a starshaped set $\Omega_{A}(S) \oplus \mathbb{B}_{\epsilon} \in \mathcal{S}_{0}\left(\mathbb{R}^{n}\right)$ which represents a robust ultimate bounded set for the system (9). This robust ultimate bounded is not dependent on the initial state.

Proof: The start-shaped property follows from the fact that the Minkowski sum is a closed operation over the class of starshaped sets (see [18]). On one hand, the ball $\mathbb{B}_{\epsilon}$ is convex, contains the origin and thus is star-shaped at the origin. On the other hand $\Omega_{A}(S)$ is an infinite Minkowski sum of star shaped sets. The set $\Omega_{A}(S)$ is positive invariant with respect to (9) and by consequence the trajectories initiated from $\Omega_{A}(S)$ will remain in $\Omega_{A}(S)$ at all future instances according to (10). This proves the ultimate boundedness for the initial points $x_{0} \in \Omega_{A}(S)$. For the initial states $x_{0} \notin \Omega_{A}(S)$ it is sufficient to observe that the trajectories are representing a superposition of two trajectories

$$
\begin{aligned}
& x_{1}(k+1)=A x_{1}(k) \\
& x_{2}(k+1)=A x_{2}(k)+w(k)
\end{aligned}
$$

initiated at $x_{1}(0)$ and $x_{2}(0)$ respectively and satisfying $x_{0}=$ $x_{1}(0)+x_{2}(0)$. While $x_{2}(k) \in \Omega_{A}(S), \forall k \geq 0$ in virtue of

\footnotetext{
${ }^{2}$ Denoted minimal robust positive invariant set, see [2] for further definitions and related characterizations.
}

the positive invariance properties, there exists a nonnegative integer $T<\infty$ such that $x_{1}(k) \in \mathbb{B}_{\epsilon}, \forall k \geq T$ and for any fixed $\epsilon>0$ according to Assumption 1. The proof of ultimate boundedness is complete.

Based on the ultimate bounds obtained for disturbances within the star-shaped set $S \subset \mathcal{S}_{0}\left(\mathbb{R}^{n}\right)$, the homogeneity properties of the dynamics (9) can be exploited to define:

$$
\Omega_{A}(\alpha S) \times(\alpha S)=\left(\alpha \Omega_{A}(S)\right) \times(\alpha S)=\alpha\left(\Omega_{A}(S) \times S\right)
$$

where the ultimate-bound set (1st block) and the disturbance set (2nd block) are scaled by alpha. This leads to a parameterized family of ultimate bounds:

$$
\begin{aligned}
\mathcal{F}_{A}(\alpha, S) & : \quad \mathbb{R}_{+} \times \mathcal{S}_{0}\left(\mathbb{R}^{n}\right) \rightarrow \mathcal{S}_{0}\left(\mathbb{R}^{n}\right) \times \mathcal{P}_{0}\left(\mathbb{R}^{n}\right) \\
\mathcal{F}_{A}(\alpha, S) & =\alpha\left(\Omega_{A}(S) \times S\right)
\end{aligned}
$$

This parametrization simply says that given a Schur matrix $A$, for any star shaped disturbance one has a unique ultimate bounded set with respect to the LTI dynamics. The scaling of the disturbance leads to a scaling of the ultimate bound.

\section{B. Nonlinear systems with state-dependent disturbances}

The link between the parameterized family of ultimate bounds for LTI systems and the ultimate bounds for the nonlinear dynamics (4) can be drawn via the transformation (7) by defining the function:

$$
\begin{array}{lll}
\mathcal{G}_{A}(\alpha, S) & : & \mathbb{R}_{+} \times \mathcal{S}_{0}\left(\mathbb{R}^{n}\right) \rightarrow \mathcal{S}_{0}\left(\mathbb{R}^{n}\right) \times \mathcal{P}_{c l}\left(\mathbb{R}^{n}\right) \\
\mathcal{G}_{A}(\alpha, S) & =\left(\alpha \Omega_{A}(S)\right) \times \tilde{W}\left(\alpha \Omega_{A}(S)\right)
\end{array}
$$

and its comparison with the counterpart in (14).

Theorem 2 Consider a star-shaped-set $S \in \mathcal{S}_{0}\left(\mathbb{R}^{n}\right)$. The set $\Omega_{A}(S) \oplus \mathbb{B}_{\epsilon} \in \mathcal{S}_{0}\left(\mathbb{R}^{n}\right)$ is an ultimate bound for (4) if

$$
\mathcal{F}_{A}(\alpha, S) \supseteq \mathcal{G}_{A}(\alpha, S), \forall \alpha \geq 1 \text {. }
$$

Proof: The set $S$ being fixed, it characterizes uniquely the set $\Omega(S)$ and consequently the ultimate bounds for a LTI system in terms of $\Omega(S) \oplus \mathbb{B}_{\epsilon}$. In the same time, by multiplying with the scalar $\alpha \in \mathbb{R}_{+}$, one obtains the set $\alpha \Omega(S)$ which preserves the invariance and leads to ultimate-boundedness properties for an LTI systems with additive disturbances within $\alpha S$. With respect to the nonlinear system (4), the set $\alpha \Omega(S) \oplus \mathbb{B}_{\epsilon}$ represent a candidate ultimate bound. By writing the nonlinear dynamics in the equivalent form (6), the link to the LTI case is established and the validity of the ultimate bounds is related to the covering of the admissible disturbance set $\tilde{W}(x)$ by $\alpha S$ with an appropriate (state-dependent) scaling $\alpha$. Two cases need to be treated in order to prove the ultimate boundedness. 

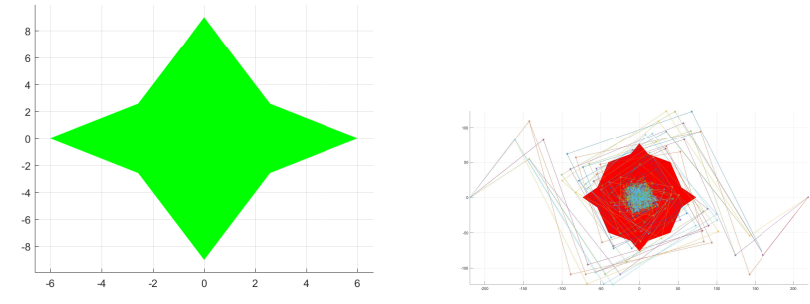

Fig. 7. A star-shaped set (left), a ultimate bound (right, red) and trajectories with random disturbances (right, colored lines)

Case 1: The invariance of $\Omega_{A}(S)$ is guaranteed if $\tilde{W}(x) \subseteq$ $S, \forall x \in \Omega_{A}(S)$. This last inclusion holds if $\tilde{W}\left(\Omega_{A}(S)\right) \subseteq S$ and is equivalent with $\mathcal{F}_{A}(1, S) \supseteq \mathcal{G}_{A}(1, S)$, i.e., the limit case $(\alpha=1)$ in (16). It follows that for the initial states $x_{0} \in$ $\Omega_{A}(S)$ the set $\Omega_{A}(S)$ itself represent an ultimate bound.

Case 2: For any initial states $x_{0} \notin \Omega_{A}(S)$, using the fact that the origin is a point in the interior of $S$, there exists $\alpha\left(x_{0}\right)>1$ such that $x_{0} \in \alpha\left(x_{0}\right) \Omega_{A}(S)$. The level of admissible additive disturbances in this particular point of the state space is represented by a set which is increasing from the origin (or more precisely from $\Omega_{A}(S)$ ). As long as the level of disturbances satisfies $\tilde{W}(x) \subseteq \alpha(x) S$, the convergence towards the ultimate bound is guaranteed based on the LTI model contractiveness. The condition $\mathcal{F}_{A}(\alpha, S) \supseteq \mathcal{G}_{A}(\alpha, S)$ resumes this requirement for all $\alpha>1$.

Example 3 Consider the system (4) with $x(k), w(k) \in \mathbb{R}^{2}$ and $f(x(k))=A_{\theta}(x(k)) x(k)$ with

$$
A_{\theta}(x(k))=0.75 *\left[\begin{array}{cc}
\cos (\theta(x(k))) & -\sin (\theta(x(k))) \\
\sin (\theta(x(k))) & \cos (\theta(x(k)))
\end{array}\right] \text {, }
$$

and $\theta(x(k))=\frac{\pi}{2}+\frac{\pi}{0.01+|x(k)|_{1}}$.

The bounds are

$W(x)=\max \left\{0.1\left(1+0.1\left|x_{1}\right|+0.1\left|x_{1} x_{2}\right|^{0.2}+0.5 \sqrt{x_{2}}\right) ; 0.9\right\} \mathcal{P}$. where $\mathcal{P}$ denotes the star-shaped set in Fig. 7 (left).

Take $A=A_{\theta}(0)$, i.e., $A$ is the Jacobian of $f$ at the origin. A star-shaped ultimate bound can be obtained readily as illustrated on the right of Fig. 7. Theorem 2 guarantees this ultimate bound to represent a ultimate bound for (4) $\forall \alpha \geq 1$.

\section{EXTENSIONS}

A. Parametrization of ultimate bounds with respect to the fixed-points

Although the focused equilibrium is the origin, the nonlinear state-dependent bounds $W(k)$ does not have to be centered at zero. Indeed, introducing flexibility in choosing the center of the disturbance set can often reduce conservativeness of computed ultimate bounds. This section will point to the structural properties of the parametrization and provide the formal extension to non-zero centered disturbances.

One of the structural properties of the results presented in the previous section is that a given star-shaped set $S \in \mathcal{S}_{0}\left(\mathbb{R}^{n}\right)$ bounding the disturbances for LTI dynamics (9) induces a function for admissible state-dependent disturbances:

$$
\begin{aligned}
& \phi(x) \quad: \quad \mathbb{R}^{n} \rightarrow \mathcal{S}_{0}\left(\mathbb{R}^{n}\right) \times \mathcal{S}_{0}\left(\mathbb{R}^{n}\right) \\
& \phi(x)=\mathcal{F}\left(\mathcal{H}_{\Omega_{A}(S)}(x), S\right)
\end{aligned}
$$

which is increasing from zero. This function, defined for each point in the state space, provides the pair of admissible disturbances and the related ultimate bound.

Although the origin is a fixed point for both the nonlinear system (4) and the linear system (9), the function

$$
\begin{aligned}
& \gamma(x): \mathbb{R}^{n} \rightarrow \mathcal{S}_{0}\left(\mathbb{R}^{n}\right) \times \mathcal{P}_{c l}\left(\mathbb{R}^{n}\right) \\
& \gamma(x)=\mathcal{G}\left(\mathcal{H}_{\Omega_{A}(S)}(.), \tilde{W}\left(\Omega_{A}(S)\right)\right)
\end{aligned}
$$

is not necessarily increasing from the origin. This makes a major difference between the linear (homogeneous) construction and the original nonlinear form (4) leading to possible conservative results whenever the Theorem 2 is used. The mitigation of a part of this conservatism represents the motivation for introducing a supplementary degree of freedom for the parametrization of the ultimate bounds inherited from the linear dynamical models. The idea will be to relax the hypothesis of a disturbance set centered at the origin and to admit an arbitrary point in the kernel of the star-shaped set.

Consider the generalized counterpart of the function (14):

$$
\begin{aligned}
\mathcal{F}_{A}(\alpha, S): & \mathbb{R}_{+} \times \mathcal{S}_{\breve{w}}\left(\mathbb{R}^{n}\right) \rightarrow \mathcal{S}_{(I-A)^{-1} \check{w}}\left(\mathbb{R}^{n}\right) \times \mathcal{S}_{\check{w}}\left(\mathbb{R}^{n}\right) \\
\mathcal{F}_{A}(\alpha, S)= & \left((I-A)^{-1} \check{w} \oplus \alpha \Omega_{A}(S \oplus\{-\check{w}\})\right) \\
& \times(\check{w} \oplus \alpha(S \oplus\{-\check{w}\}))
\end{aligned}
$$

and the generalized function with respect to (15):

$$
\begin{aligned}
\mathcal{G}_{A}(\alpha, S): & \mathbb{R}_{+} \times \mathcal{S}_{\breve{w}}\left(\mathbb{R}^{n}\right) \rightarrow \mathcal{S}_{(I-A)^{-1} \check{w}}\left(\mathbb{R}^{n}\right) \times \mathcal{P}_{c l}\left(\mathbb{R}^{n}\right) \\
\mathcal{G}_{A}(\alpha, S)= & \left((I-A)^{-1} \check{w} \oplus \alpha \Omega_{A}(S \oplus\{-\check{w}\})\right) \\
& \times \tilde{W}\left((I-A)^{-1} \check{w} \oplus \alpha \Omega_{A}\left(S_{\breve{w}} \oplus\{-\check{w}\}\right)\right)
\end{aligned}
$$

then the following result resumes the sufficient conditions for the existence of ultimate bounds with respect to the non-linear dynamics.

Theorem 3 Consider $S$, a star-shaped-set in $\mathbb{R}^{n} . \Omega_{A}(S) \oplus$ $\mathbb{B}_{\epsilon}$ is an ultimate bound for (4) if there exists a point $\check{w} \in$ $\operatorname{Int}(\operatorname{kern}(S))$ such that $\mathcal{F}_{A}\left(\alpha, S_{\breve{w}}\right) \supseteq \mathcal{G}_{A}\left(\alpha, S_{\breve{w}}\right), \forall \alpha \geq 1$.

Proof: Similar to Theorem 2. 


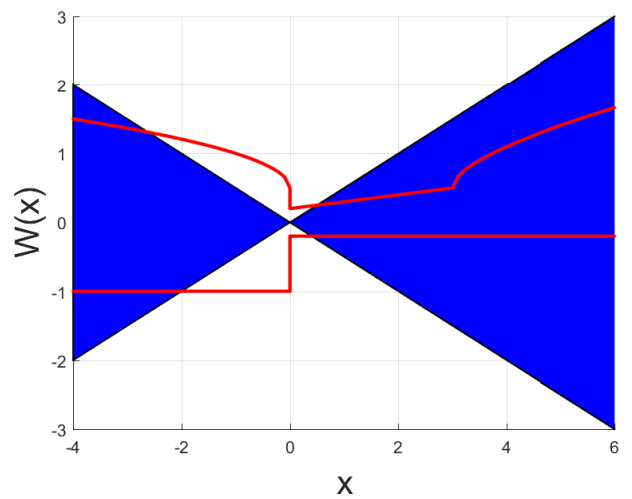

Fig. 8. Validation of the sufficient condition in Theorem 2 for $\mathcal{F}_{A}(\alpha, S)$ depicted in blue, based on a set centered at the origin $S=[-1,1] \in \mathcal{S}_{0}\left(\mathbb{R}^{n}\right)$. The state dependent bounds are depicted in red.

Example 4 In order to illustrate the reduction of conservativeness in the ultimate bound estimation let us consider the system:

$$
x(k+1)=0.5 x(k)+w(k)
$$

with state-dependent bounds on the disturbance:

$$
\begin{array}{ll}
-1 \leq w(x) \leq 0.5+0.5 \sqrt{x} & \text { if } x \leq 0 \\
-0.2 \leq w(x) \leq 0.2+0.1 x & \text { if } 0 \leq x \leq 3 \\
-0.2 \leq w(x) \leq 0.1(2+x+5 * \sqrt{x-3}) & \text { if } x \geq 3
\end{array}
$$

The parametrization of invariant sets is particularly simple:

$$
\mathcal{F}_{A}(\alpha, S)=\alpha((2 S) \times S) .
$$

By considering $S=[-1,1]$, the sufficient condition in Theorem 2 leads to the ultimate bound $|x| \leq 2.618$ shown in Fig. 8.

By using $\check{w}=0.2$ in Theorem 3, the symmetry with respect to the origin is relaxed and the ultimate bounds can be improved up to

$$
|x| \leq 2.02
$$

as shown in Fig. 8.

\section{B. Active input design and impact on the ultimate-bounds}

Based on the previous discussion, the parametrization can be utilized for active input design. By considering the system with both disturbances and inputs:

$$
x(k+1)=f(x(k))+w(k)+\bar{u},
$$

the natural idea is to consider the case where the exogenous signal can be complemented with a feed-forward control $w_{1}(k)=w(k)+\check{u}$ thus playing an active role in the design of improved ultimate bounds upon the theoretical foundations of the Theorem 2. Finally, once the system trajectory enter the

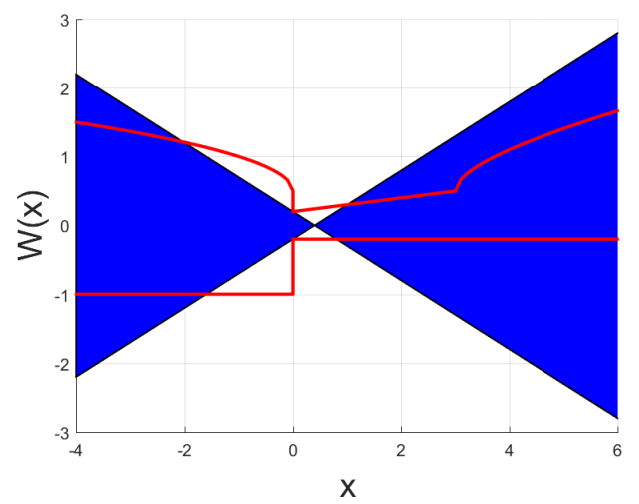

Fig. 9. Validation of the sufficient condition in Theorem 3 for $\mathcal{F}_{A}(\alpha, S)$ depicted in blue, based on a set $S=[-0.8,1.2] \in \mathcal{S}_{0.2}\left(\mathbb{R}^{n}\right)$. The state dependent bounds are depicted in red.
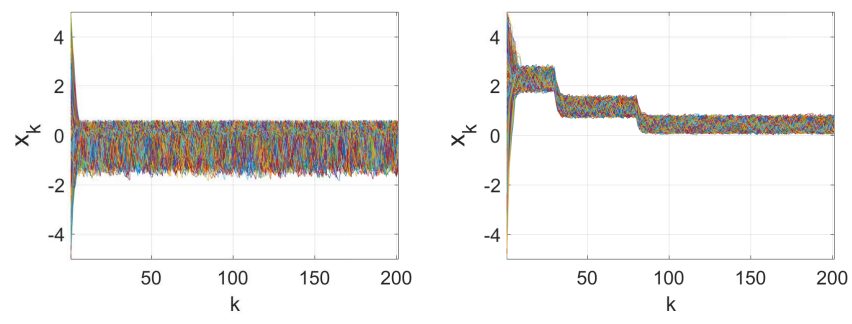

Fig. 10. Superposition of 1000 trajectories of (23) generated with different initial conditions and random disturbance realizations within the bounds. Left: absence of an active control signal $u(k)=0, \forall k$. Right: piecewise constant active input (25).

ultimate bounds, the input signal can be updated in order to further adjust the shape of the ultimate bounds thus leading to a feedback scheme

$$
w_{2}(k)=w(k)+u(k)
$$

To illustrate the concept, consider again Example 4. In the absence of an active input, the system converge towards an ultimate bound presented in the left part of Fig. 10. The piecewise constant input signal:

$$
u(k)=\left\{\begin{array}{c}
1 \text { for } 0 \leq k \leq 30 \\
0.5 \text { for } 30 \leq k \leq 80 \\
0.18 \text { for } 80 \leq k
\end{array}\right.
$$

leads to the result presented in the right hand side of Fig. 10 with obvious improvements in terms of ultimate bounds.

These design avenues are subject to current developments, the algorithmic part of the developments being of primer importance, according to the topology to the set-valued maps in Assumption 2. For example, to achieve a desired small ultimate bound, a judicious switching procedure is needed, according to the assumptions, in order to make sure that 
equilibria are not in the exterior of the ultimate set in the presence of $w_{i}$.

\section{Conclusions}

A novel framework for the analysis of systems affected by additive state-dependent disturbances has been introduced based on set-theoretic notions. It aims to relax the assumptions on the bounds and mappings as for example to deal with systems which have asymmetric nonlinearities. The basic principle is to make use of a linear nominal part for which the ultimate bounds exist. The ultimate bounds of the whole nonlinear system are parameterized in terms of shapes and scaling factors of the disturbances representing nonlinearities. It is worth to be mentioned that star-shaped sets are employed within the parametrization thus avoiding the usual convexity assumptions of the linear analysis. Several sufficient conditions are presented for the characterization of ultimate bounds, with details on the degrees of freedom within the parametrization. The approach is considered attractive for the design of openloop or closed-loop excitation signals to improve the ultimate bounds.

\section{REFERENCES}

[1] V. M. Kuntsevich and B. N. Pshenichnyi, "Minimal invariant sets of dynamic systems with bounded disturbances," Cybernetics and Systems Analysis, vol. 32, no. 1, pp. 58-64, 1996.

[2] I. Kolmanovsky and E. G. Gilbert, "Theory and computation of disturbance invariant sets for discrete-time linear systems," Mathematical problems in engineering, vol. 4, no. 4, pp. 317-367, 1998.

[3] K. Hirata and Y. Ohta, " $\epsilon$-feasible approximation of the state reachable set for discrete-time systems," in Decision and Control, 2003. Proceedings. 42nd IEEE Conference on, vol. 5. IEEE, 2003, pp. 5520-5525.

[4] S. V. Rakovic, E. C. Kerrigan, K. I. Kouramas, and D. Q. Mayne, "Invariant approximations of the minimal robust positively invariant set," IEEE Trans. Automatic Ctrl., vol. 50, no. 3, pp. 406-410, 2005.

[5] C.-J. Ong and E. G. Gilbert, "The minimal disturbance invariant set: Outer approximations via its partial sums," Automatica, vol. 42, no. 9, pp. 1563-1568, 2006.

[6] F. Blanchini and S. Miani, Set-theoretic methods in control. Springer, 2008.

[7] V. Reppa, M. M. Polycarpou, C. G. Panayiotou et al., "Sensor fault diagnosis," Foundations and Trends in Systems and Control, vol. 3, no. 1-2, pp. 1-248, 2016.

[8] D. Q. Mayne, M. M. Seron, and S. Raković, "Robust model predictive control of constrained linear systems with bounded disturbances," $A u$ tomatica, vol. 41, no. 2, pp. 219-224, 2005.

[9] J. B. Rawlings and D. Q. Mayne, Model predictive control: Theory and design. Nob Hill Pub., 2009.

[10] K. H. Khalil, "Nonlinear systems.” 3rd ed., Prentice Hall, 2002.

[11] F. Blanchini, "Ultimate boundedness control for uncertain discrete-time systems via set-induced Lyapunov functions," in Decision and Control, 1991., IEEE Conf. on, 1991, pp. 1755-1760.

[12] E. Kofman, H. Haimovich, and M. M. Seron, "A systematic method to obtain ultimate bounds for perturbed systems," International Journal of Control, vol. 80, no. 2, pp. 167-178, 2007.
[13] S. Olaru and V. Reppa, "Ultimate bounds and robust invariant sets for linear systems with state-dependent disturbances," in Developments in Model-Based Optimization and Control. Springer, 2015, pp. 339-359.

[14] E. D. Sontag, "Input to state stability: Basic concepts and results," in Nonlinear and optimal control theory. Springer, 2008, pp. 163-220.

[15] Z.-P. Jiang and Y. Wang, "Input-to-state stability for discrete-time nonlinear systems," Automatica, vol. 37, no. 6, pp. 857-869, 2001.

[16] Z.-P. Jiang, A. R. Teel, and L. Praly, "Small-gain theorem for ISS systems and applications," Mathematics of Control, Signals and Systems, vol. 7, no. 2, pp. 95-120, 1994.

[17] H. Ito, "Utility of iISS in composing lyapunov functions for interconnections," IFAC Proceedings, vol. 46, no. 23, pp. 723-730, 2013.

[18] S. Olaru, J. De Doná, M. Seron, and F. Stoican, "Positive invariant sets for fault tolerant multisensor control schemes," International Journal of Control, vol. 83, no. 12, pp. 2622-2640, 2010. 